\title{
RESEARCH PAPER \\ DEVELOPMENT AND EVALUATION OF LYOPHILIZED THIOLATED-CHITOSAN WAFERS FOR BUCCAL DELIVERY OF PROTEIN
}

\author{
I. Ayensu ${ }^{1,2}$ and J. S. Boateng ${ }^{2}$ \\ ${ }^{I}$ Department of Pharmaceutical, Chemical and Environmental Sciences, School of Science, \\ University of Greenwich at Medway, Chatham Maritime, Kent, UK, ME4 4TB \\ i.ayensu@gre.ac.uk \\ ${ }^{2}$ Department of Pharmaceutical Chemistry, Faculty of Pharmacy and Pharmaceutical Sciences, \\ KNUST, Kumasi \\ j.s.boateng@gre.ac.uk
}

\begin{abstract}
Annealed and non-annealed freeze-dried wafers from synthesised thiolated-chitosan have been developed and evaluated. Wafers were obtained by freeze-drying aqueous gels of the thiolated polymer incorporating per polymer weight, $10 \%$ each of glycerol as plasticizer, D-mannitol as cryoprotectant and 50\% BSA as model protein drug. The formulation was freeze-dried with or without the process of annealing. Texture analyzer was employed to investigate the in vitro mucoadhesive properties in tensile mode, residual moisture content by thermo-gravimetric analysis (TGA) while hydration capacity and drug release studies were performed in 0.1M PBS. Microscopic architecture was examined using scanning electron microscopy (SEM). Differential scanning calorimetry (DSC) was employed for protein phase separation studies and conformational stability by attenuated total reflectance-Fourier transform infra-red spectroscopy (ATR-FTIR) and confirmed with Circular dichroism (CD). The annealing process led to wafers with increased ease of hydration, improved in vitro mucoadhesive characteristics and enhanced BSA release, without affecting the conformational stability of the protein due to the presence of a cryoprotectant. These results show the potential application of annealed freeze-dried thiolatedchitosan wafers for buccal mucosa delivery of protein based drugs.
\end{abstract}

Keywords: Thiolated-chitosan, freeze-drying, annealing, buccal delivery, protein

\section{INTRODUCTION}

Chitosan (CS) (poly[-(1,4)-2-amino-2-deoxy-D -glucopiranose]) is a natural polymer obtained by deacetylation of chitin (Wu et al., 2009). Due to its biocompatibility and biodegradation properties (VandeVord et al., 2002), chitosan has been used primarily for the purpose of improved mucoadhesion, permeation enhancement and drug delivery (Agnihotri et al., 2004) via mucosal routes such as GI mucosa (Van Der Lubben et al., 2001; Read et al., 2005), buccal (Giunchedi et al., 2002), and ocular (De Campos et al., 2004).

The mucoadhesive properties exhibited by chitosan have been further improved by derivatization to generate thiolated-chitosans. Factors that can alter the interaction between the polymer 
and the mucosal layer can affect the mucoadhesive property. These include polymer molecular weight (Andrew et al., 2009), polymer concentration (Solomonidou et al., 2001) and drying method. Freeze-dried formulations offer stable products, extend shelf-life and allows storage of products at room temperature (Bunte et al., 2010). However, during the freezing stage of freeze-drying, low molecular weight components tend to undergo incomplete crystallization resulting in the formation of mixtures of different polymorphs (Liao et al., 2007). Such processes may cause reproducibility and product characterization problems. Annealing may be employed to foster crystallization of the ingredients and to improve pore size of ice crystals (Searles et al., 2001a).

This report describes the development and evaluation of the functional characteristics of freeze-dried annealed and non-annealed thiolated-chitosan wafers, as potential drug delivery systems via the buccal mucosa. The wafers were characterised for moisture content, mucoadhesion properties and microscopic structure using thermo-gravimetric analysis (TGA), texture analysis (TA) and scanning electron microscopy (SEM) respectively. The conformational stability of bovine serum albumin (BSA) was studied with attenuated total reflectance-Fourier transform infra-red spectroscopy (ATR-FTIR) and circular dichroism (CD). The effect of annealing on hydration, mucoadhesion and the release of BSA from the formulations were also evaluated.

\section{MATERIALS AND METHODS \\ Materials}

Chitosan (medium molecular weight, 190-310 $\mathrm{kDa}, 75-85 \%$ deacetylated), BSA (mol wt $66 \mathrm{kDa}$ ), glycerol, D-mannitol, Bradford reagent, thioglycolic acid (TGA), N-(3dimethylaminopropyl)-N-ethyl carbodiimide hydrochloride (EDAC), L-cysteine hydrochloride, 5,5'-dithiobis (2-nitrobenzoic acid) (Ellman's reagent) and mucin (from bovine submaxillary gland, Type I-S) were all obtained from Sigma (Gillingham, UK). Pulverised gela- tine was purchased from Fischer Scientific (Loughborough, UK). All other reagents were of analytical grade and were used without further purification.

\section{Chitosan-4-thioglycolic acid (CS-TGA) syn- thesis and purification}

Synthesis of CS-TGA was by method previously described (Saboktakin et al., 2011). 500 $\mathrm{mg}$ of CS was dissolved in $50 \mathrm{~mL}$ of $0.1 \mathrm{M}$ $\mathrm{HCl}$. An equivalent of $500 \mathrm{mg}$ of thioglycolic acid (TGA) was added followed by $50 \mathrm{mM}$ EDAC in order to activate the carboxylic acid moieties of TGA, the $\mathrm{pH}$ adjusted to 5 with $1 \mathrm{M}$ $\mathrm{NaOH}$ at room temperature and stirred for 3 hours. Purification to remove unconjugated TGA and sulfhydryl immobilization determination of CS-TGA was by the method reported in literature (Bernkop-Schnurch et al., 2004), using Float-A-Lyzer G2 dialysis device (8-10 kDa Molecular weight cut-off, Sigma Gillingham, UK) and Ellman's reaction with standard cysteine $\mathrm{HCl}$ curve $\left(r^{2}>0.99\right)$ respectively.

\section{Gel formulation and freeze-drying}

Ten percent (per polymer weight) each of glycerol and D-mannitol as plasticizer and cryoprotectant respectively were added to the recovered dialysed gel and loaded with BSA as a model protein drug in a 2:1 CS-TGA: BSA ratio. The final formulated gel was stirred continuously for 30 minutes at room temperature to obtain a uniform gel which was kept under ambient conditions to remove all air bubbles.

Six grammes $(6.0 \mathrm{~g})$ of the homogeneous gel was transferred into a mould (diameter $35 \mathrm{~mm}$ ) and freeze-dried using a novel freeze-drying cycle developed with differential scanning calorimetry (DSC) (Ayensu et al., 2011) on a Virtis AdVantage XL 70 freeze dryer (Biopharma Process Systems, Winchester, UK). An annealing temperature of $-25^{\circ} \mathrm{C}$ (3 hours) was applied during the freezing phase ($55^{\circ} \mathrm{C}$ ). Primary and secondary drying were at $30^{\circ} \mathrm{C}$ and $20^{\circ} \mathrm{C}$ respectively, with a vacuum of 20 mtorr for 34 hours. The effect of annealing on the physico-mechanical properties of the 
drug loaded formulations was determined by freeze-drying with or without the process of annealing. Resultant freeze-dried wafers were stored over anhydrous silica gel till ready for characterisation.

\section{Physico-mechanical characterisation of wafers \\ SEM}

The external surfaces of the freeze-dried wafers were placed on the exposed side of a double sided carbon adhesive tape on labelled stainless steel stubbs and sputter coated with gold for 120 seconds at $1 \mathrm{kV}$ and 30mA (Edwards Sputter Coater S150B). They were then placed in the chamber of a Cambridge Stereoscan S-360 SEM (Class one equipment, London UK). Images were processed with i-scan2000 software after acquisition at an accelerating voltage of $20 \mathrm{KV}$ and a working distance of $15 \mathrm{~mm}$.

\section{Swelling capacity}

The wafers were initially weighed and the swelling behaviour observed at predetermined time intervals (Wu et al., 2009) by incubation at $37 \pm 0.1^{\circ} \mathrm{C}$ in $25 \mathrm{~mL}$ of $0.1 \mathrm{M}$ PBS solution $(\mathrm{pH} 6.8 \pm 0.1$ simulating salivary $\mathrm{pH})$. The samples were removed and blotted off carefully between tissue papers to remove the surfaceadhered liquid droplets and reweighed on an electronic balance to a constant weight. The percentage of water uptake was calculated as follows:

Swelling capacity $(\%)=100 \times(P i-P) / P .$.

where $P i$ is the weight of the hydrated wafer, and $P$ is the initial weight of wafer.

\section{Moisture content}

The estimation of percentage residual moisture of the freeze-dried wafers was by Thermal Advantage TGA 2950 (TA Instruments, Crawley UK) (Wang, W. 2000). Wafers weighing between $3-6 \mathrm{mg}$ were placed in a previously tared $70 \mu \mathrm{l}$ aluminium crucible and a constant phase of heating at $15^{\circ} \mathrm{C} / \mathrm{min}$ from ambient temperature to a maximum of $150{ }^{\circ} \mathrm{C}$ was applied with a constant stream of dry nitrogen. The percentage weight loss from a second derivative plot was determined using TA Universal Analysis 2000 programme.

\section{In vitro mucoadhesion studies}

Mucoadhesive measurements were performed on the freeze-dried annealed and non-annealed CS-TGA-BSA wafers using our previously reported method (Ayensu et al. 2011). Briefly, samples $(n=4)$ were attached to a $75 \mathrm{~mm}$ diameter adhesive rig probe with a double sided adhesive tape on a TA.HD.plus Texture Analyser (Stable Micro Systems, Surrey, UK) fitted with a $5 \mathrm{~kg}$ load cell in tension mode. A set gelatine gel in a Petri-dish (diameter $88 \mathrm{~mm}$ ) was used to represent the buccal mucosal surface for the in vitro measurement. The model mucosal substrate was equilibrated with $2 \%$ mucin solution (adjusted to $\mathrm{pH}$ of $6.8 \pm 0.1$ ) for 60 seconds and placed on the instrument platform. The probe, lined with wafer was set to approach the model mucosal surface with pretest speed of $0.5 \mathrm{~mm} / \mathrm{s}$, test speed of $0.5 \mathrm{~mm} / \mathrm{s}$ and post speed of $1.00 \mathrm{~mm} / \mathrm{s}$. The Texture Exponent 32 software was employed to record and process the data. The maximum force required to detach the wafer on the upper probe from the mucosal surface, known as the peak adhesive (PAF) was determined. The area under the curve (AUC) representing total work of adhesion (TWA) of the wafers was estimated from the force-distance plot whiles the cohesiveness of the samples was determined by the distance of travel. The influence of annealing on mucoadhesive strength of the thiolated wafers was investigated.

\section{In vitro $B S A$ release profiles}

The freeze-dried wafers were immersed in beakers containing $50 \mathrm{ml}$ of $0.1 \mathrm{M}$ PBS $(\mathrm{pH} 6.8$ $\pm 0.1)$ solution as release medium at $37 \pm 0.1^{\circ}$ C, covered (Nochos et al., 2008) and stirred at $150 \mathrm{rpm}$ with a magnetic stirrer. Fifty $\mu \mathrm{L}$ of each sample was withdrawn at predetermined time intervals, replaced with the same amount of the release medium to maintain a constant volume for 7 hours and treated with $1 \mathrm{~mL}$ Brad- 
ford's reagent and the absorbance measured at 595nm and 450nm (Ernst and Zor, 2010). A linearised calibration curve $\left(r^{2}>0.99\right)$ was used to determine the concentration of the BSA released and the corresponding cumulative percentage drug release profiles of BSA were then plotted.

\section{Stability of protein in freeze-dried wafers DSC}

The freeze-dried CS-TGA wafers were studied for possible phase separation after freezedrying on the DSC. This involved the initial cooling on the DSC at $10{ }^{\circ} \mathrm{C} / \mathrm{min}$ from 25 to $50{ }^{\circ} \mathrm{C}$. It was then heated at a rate of $20^{\circ} \mathrm{C} / \mathrm{min}$ to $200{ }^{\circ} \mathrm{C}$ and the cycle repeated. An empty aluminium pan was used as reference. Mettler STARe software was used to analyse and evaluate the DSC thermograms.

\section{ATR- FTIR analysis}

ATR-FTIR spectra of the samples were acquired on an Excalibur series FTS 3500 ARX FTIR, equipped with Specac Golden gate (Varian, Oxford UK). Win IR PRO software of wave number range $600-4000 \mathrm{~cm}^{-1}$ was used for the analysis. The spectra were collected at a resolution of $1.5 \mathrm{~cm}^{-1}$ with 16 scans per spectrum. A background spectrum was acquired and assigned for use on subsequent spectral acquisitions for each sample.

\section{Conformational stability of BSA-CD}

The secondary structure of BSA in the freezedried wafers was examined using far-UV CD. Spectrum of a $0.5 \mathrm{mg} / \mathrm{mL}$ solution of native BSA dissolved in $0.1 \mathrm{M}$ PBS $(\mathrm{pH} 6.8)$ as the negative control or BSA released from the freeze-dried wafers was recorded at $25^{\circ} \mathrm{C}$ from wavelength range $240 \geq \lambda \geq 190$ with bandwidth $1 \mathrm{~nm}$, time-per-point $0.5 \mathrm{~s}$, and cell pathlength of $0.1 \mathrm{~mm}$ using $\mathrm{CD}$ spectroscopy (Chirascan, Applied Photophysis, Surrey, UK) . The mean residue ellipticity $[\theta]$ mrw, $\mathrm{k}$ was determined by:

$$
[\theta] \mathrm{mrw}, \mathrm{k}=\quad \text { MRW } \times \theta_{\mathrm{\kappa}} / 10 \times \mathrm{d} \mathrm{x} \mathrm{c}
$$

where $\theta \mathrm{k}$ is the observed ellipticity (degrees) at wavelength $\mathrm{k}, d$ is the pathlength $(\mathrm{cm})$, and $c$ is the concentration $(\mathrm{g} / \mathrm{mL})$ (Kelly et al., 2005).

\section{Statistical evaluation}

Statistical evaluation of data was carried out using two tailed student t-test with $95 \%$ confidence interval as the minimal level of significance.

\section{RESULTS AND DISCUSSION}

\section{Chitosan-4-thioglycolic acid (CS-TGA) syn-} thesis and purification

In the present study, TGA was bound to CS via amidation between the carboxylic group of TGA and the primary amino groups of CS. The reactivity of the primary amino group at the 2position of glucosamine subunit of chitosan is exploited for the immobilization of thiol groups. The process was performed at a $\mathrm{pH} \leq 5$ to prevent the accidental formation of disulphide bonds by air oxidation during synthesis. The reactive thiolate anion concentration for the oxidation of thiol groups is low at this $\mathrm{pH}$, which prevents the formation of disulphide bonds. Ellman's reagent was employed in the determination of the amount of reduced and oxidized thiol groups immobilized (Hornof et al., 2003) without previous quantitative reduction of disulfide bonds with borohydride. The CS-TGA conjugate exhibited $236 \pm 26 \mu \mathrm{mol}$ thiol groups per gram of polymer. A degree of modification of 25-250 mmol thiol groups per gram of chitosan leads to the highest improvement in the mucoadhesive and permeation enhancing properties of these thiolated chitosans (Sreenivas and Pai, 2009).

\section{Gel formulation and freeze-drying}

Flexible, tough, non-brittle and porous freezedried annealed and non-annealed CS-TGABSA wafers were produced with medium molecular weight chitosan as the base matrix. The primary drying phase of the freeze-drying cycle was deemed complete when the product temperature measured by means of probes equalled the shelf temperature of $-30{ }^{\circ} \mathrm{C}$ after 14 hours. 
Annealing as a process step maintains samples at a specified temperature below the equilibrium freezing point but above the glass transition temperature. The incorporation of an annealing process helps to achieve faster water vapour transport, shorter primary drying time and elegant porous wafers (Searles et al., 2001b). This allows for the formation of larger ice crystals resulting in increased sublimation rate during the primary drying stage, creating larger pores in the process. In addition, the process of annealing facilitates crystallisation of active ingredients and the cryoprotectant (mannitol). This promotes solute and ice crystal growth and prevents premature crystallisation of metastable glass such as D-mannitol.

\section{Physico-mechanical characterisation of wafers \\ SEM}

SEM was used to study the pore size and structure of the freeze-dried wafers (morphology). Figure 1 shows representative micrographs of the freeze-dried wafers. The micrographs from the SEM show the effect of annealing on the pore size. Annealing promotes the merging of smaller ice crystals to form larger ones, the sublimation of which creates larger pores. The wafers formed an interconnecting network of polymeric circular pores, with comparable pore size distribution. The annealed CS-TGA-BSA wafers however, formed more fibrous spongy-

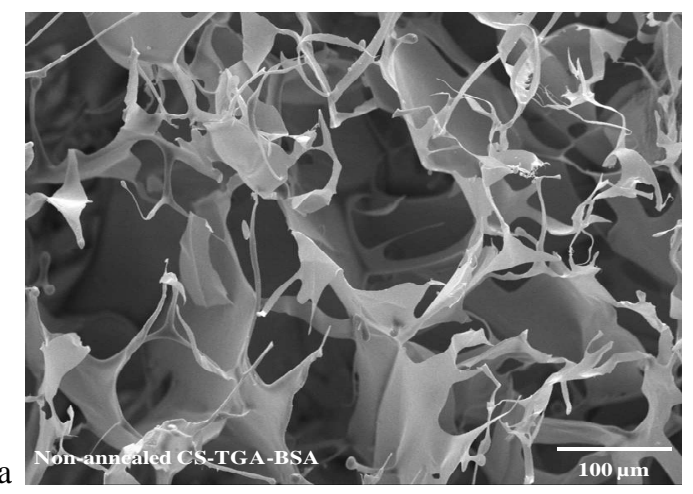

like porous network.

\section{Swelling capacity}

The difference in the swelling capacities of the annealed and the non-annealed CS-TGA-BSA wafers (Table 1) was statistically significant ( $\mathrm{p}$ $<0.003$ ). It is worth noting that the annealed wafers maintained their structural integrity during the incubation period ( 4 hours) due to the stronger mechanical structure. This was not the case for the non-annealed wafers which formed loose jelly-like and rather unstable swollen structures that consequently disintegrated upon removal from the hydration medium after two (2) hours of incubation at $37^{\circ} \mathrm{C}$.

\section{Moisture content}

The residual moisture contents of the freezedried wafers are shown in Table 1. The annealed wafers had a slightly lower residual moisture content compared to the non-annealed products; however, no significant statistical difference was observed $(p>0.3)$. The loss in weight observed for all the samples during the constant heating stage occurred between $60{ }^{\circ} \mathrm{C}$ and $120{ }^{\circ} \mathrm{C}$, an indication of the fact that weight loss was due to bound water. Attaining adequate residual moisture content for wafers is vital as lower water content reduces molecular mobility and increases shelf-life by avoiding premature hydration of the active protein drug (Bunte et al., 2010).

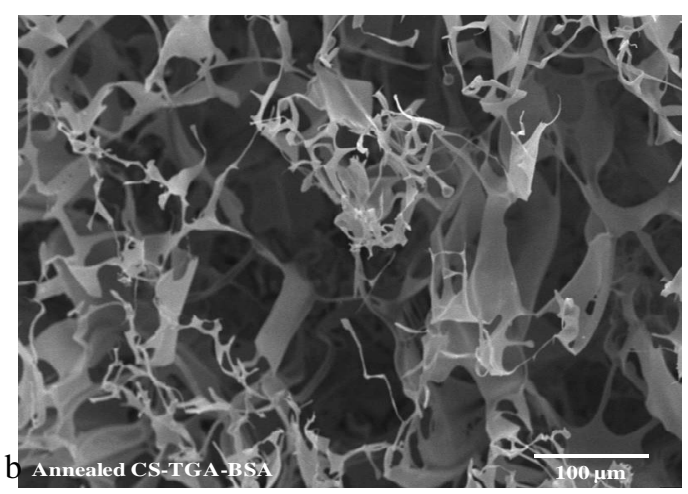

Fig. 1: Representative SEM micrographs showing annealed and non-annealed CS-TGA-BSA wafers ((a) Non-annealed CS-TGA-BSA (b) Annealed CS-TGA-BSA) 
Table 1: Percentage drug loading capacity, moisture content and hydration capacity of wafers

\begin{tabular}{lcc}
\hline Sample & $\begin{array}{c}\text { \% Moisture content } \\
(\boldsymbol{n}=\mathbf{4}, \text { mean } \pm \boldsymbol{S D})\end{array}$ & $\begin{array}{c}\text { \% swelling capacity } \\
(\boldsymbol{n}=\mathbf{4}, \text { mean } \pm \boldsymbol{S D})\end{array}$ \\
\hline $\begin{array}{l}\text { Annealed } \\
\text { CS-TGA-BSA }\end{array}$ & $1.7 \pm 0.1$ & $482 \pm 18.2$ \\
$\begin{array}{l}\text { Non Annealed } \\
\text { CS-TGA-BSA }\end{array}$ & $1.9 \pm 0.4$ & $434.0 \pm 3.1$ \\
\hline
\end{tabular}

\section{In vitro mucoadhesion studies}

The process of annealing led to significant differences in the mucoadhesive properties of the CS-TGA wafers. The annealed CS-TGA-BSA wafers showed significant improvement in mucoadhesive properties (Fig. 2) compared to the non-annealed CS-TGA-BSA wafers. The annealed CSTGA-BSA wafers with enhanced swelling capacity had higher PAF and cohesiveness compared to the non-annealed CSTGA-BSA wafers. The total work of adhesion (TWA) further showed a statistically significant difference $(p<0.0001)$ between the annealed and non annealed CS-TGA-BSA wafers, with an approximately 4-fold increase in mucoadhesive strength. The major differences observed in the PAF, TWA and cohesiveness of the wafers were largely due to the increased mucoadhesive strength impacted by the mechanically strong and fibrous porous structure of the annealed wafers.

\section{In vitro BSA release profiles}

The dissolution profiles of BSA from the freeze -dried wafers in 0.1M PBS is shown in Fig. 3. The total cumulative percent BSA release from the annealed and non annealed CS-TGA-BSA CS-BSA were $95.2 \pm 7.3 \%$ and $84.0 \pm 4.7 \%$ respectively. This difference is considered to be statistically significant $(p<0.05)$ with both formulations exhibiting sustained release profiles, typical of chitosan based formulations. The drug release may have been facilitated by the porous network of freeze-dried wafers as a result of increased surface of the dispersed drug in the porous cake which accelerates dissolu- tion significantly (Bunte et al., 2010). The total percent release was higher from the annealed CSTGA than the non annealed CS-TGA wafers due to its higher rate of hydration and enhanced cohesiveness. The cohesion and stability of a drug delivery system over the intended duration of drug release is often a requirement for controlled release (Peppas and Bury, 1985). The lower total percent release of BSA could be attributed to the reduced cohesiveness of the non-annealed wafers.

\section{Stability of protein in freeze-dried wafers DSC}

Multiple glass-transitions detected by DSC after freeze-drying indicate possible phase separation of protein in the freeze-dried wafer and/or presence of metastable crystalline structures. For phase separation studies after freezedrying, no glass-transition temperatures were detected in the freeze-dried wafers, an indication of product stability during storage.

\section{ATR- FTIR analysis}

To further investigate the secondary structure of BSA in the drug loaded wafers, ATR-FTIR spectroscopy was employed. Structural information was obtained for the protein loaded freeze-dried wafers through the analysis of the conformationally sensitive amide I band, which is located between 1600 and $1700 \mathrm{~cm}^{-1}$ (Fig. 4). This band is due to the in-plane $\mathrm{C}=\mathrm{O}$ stretching vibration, weakly coupled with $\mathrm{C}-\mathrm{N}$ stretching and in-plane $\mathrm{N}-\mathrm{H}$ bending. Each type of secondary structure (i.e. $\alpha$-helix, $\beta$-turn and disordered gives rise to a different $\mathrm{C}=\mathrm{O}$ stretching 


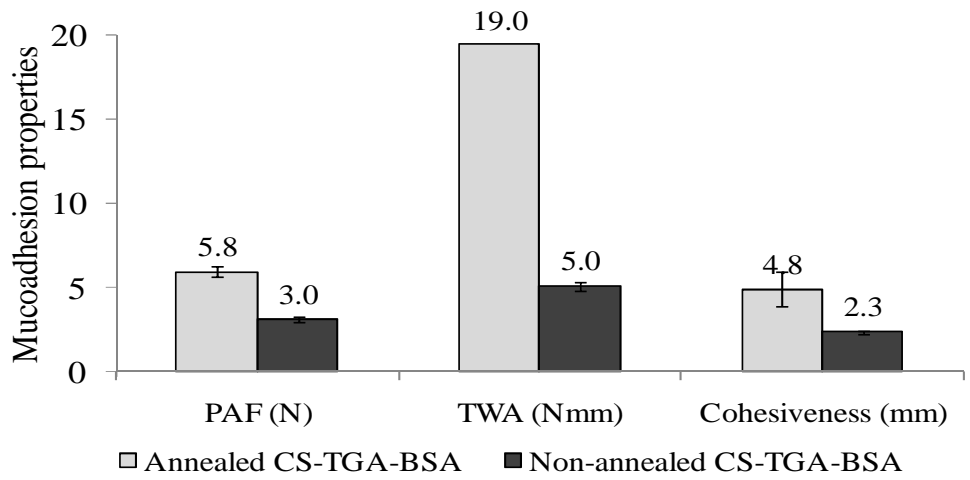

Fig. 2: In vitro mucoadhesion force measurements of wafers showing the PAF, TWA and cohesiveness $(n=4$ mean $\pm s d)$

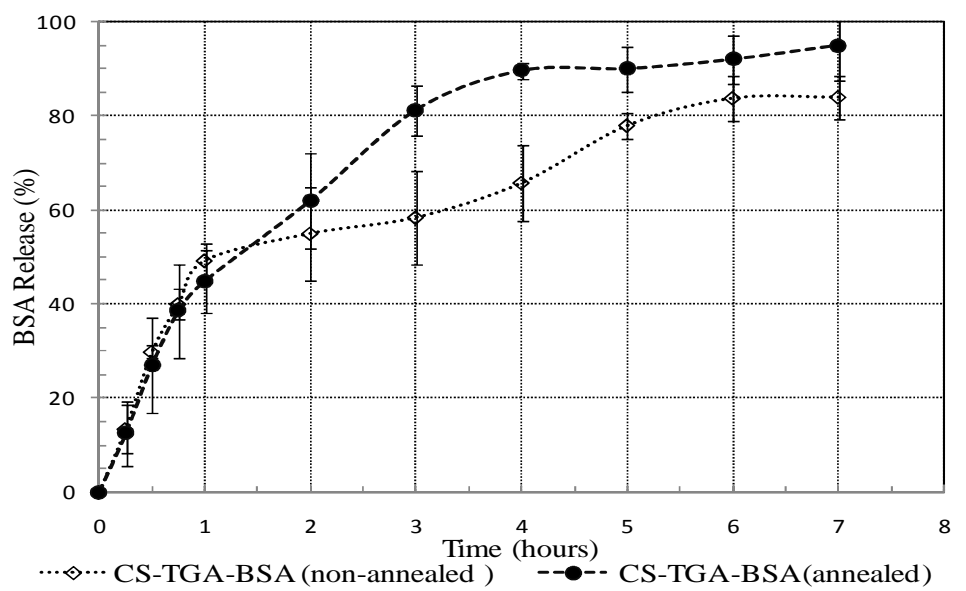

Fig. 3: Cumulative percent BSA release from annealed and non-annealed CS-TGA-BSA wafers $(n=4$ mean $\pm s d)$

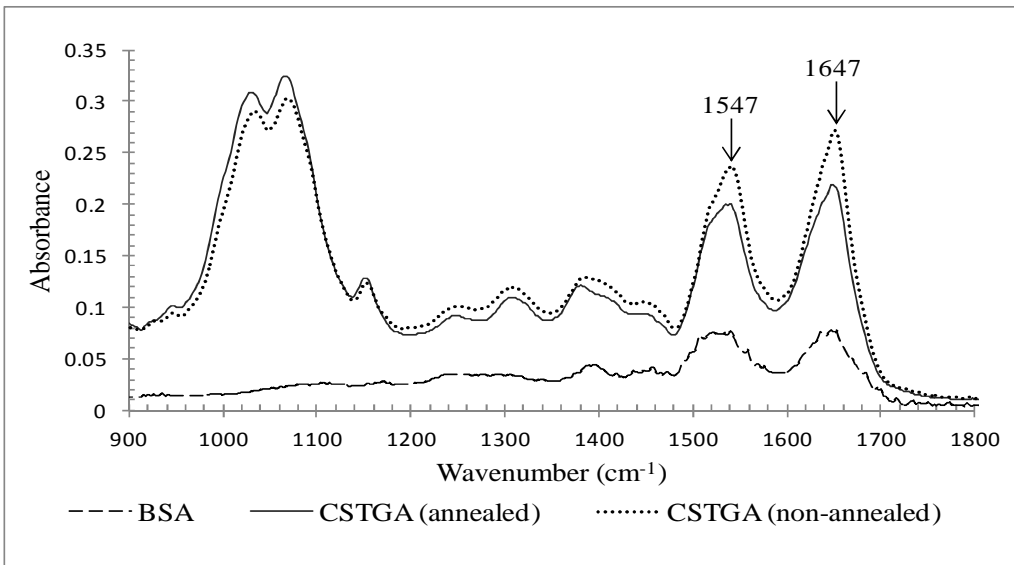

Fig. 4: ATR-FTIR Spectra of native BSA, annealed and non-annealed CS-TGA-BSA wafers 
frequency which has a characteristic band position. The ATR-FTIR spectra show that the absorption peaks of annealed and non annealed CS-TGA-BSA at $1647 \mathrm{~cm}^{-1}$ (amide I band) were stronger than those of native BSA due to the presence of chitosan. The absorption peak at $1574 \mathrm{~cm}^{-1}$ is usually attributed to amide II band (C-N stretching and N-H bend) present in both BSA and chitosan.

In all FT-IR patterns, the main peaks corresponding to $\alpha$-helical remained unchanged, suggesting that the protein structure maintained its folded conformation, and was neither degraded nor hydrolyzed during the production of freeze-dried CSTGA- BSA wafers irrespective of annealing process.

\section{Physical stability of BSA-CD}

The conformational stability of in vitro released BSA from the freeze-dried wafers was assessed by $\mathrm{CD}$. The $\mathrm{CD}$ profile of native BSA and BSA released from the wafers, both in PBS at $\mathrm{pH} 6.8$ in far UV range are shown in Fig. 5. As is evident from this figure, the native BSA had two minima around 208 and $222 \mathrm{~nm}$; the first band was assigned to $\alpha$-helical structure, while the second band was assigned to the $\beta$-structure.
These results are consistent with the crystal structure of BSA (Quiming et al., 2005). The ratios between the mean residue ellipticity at 208 and $222 \mathrm{~nm}\left([\theta]_{208} /[\theta]_{222}\right)$ were $1.10,1.11$ and 1.11 for native BSA and BSA released from the annealed and non annealed CSTGABSA respectively.

The similarity of the far-UV CD spectra and the mean residue ellipticity obtained confirmed the conformational stability of BSA in the freezedried wafers.

\section{CONCLUSION}

The various techniques used to characterise the freeze-dried annealed and non annealed thiolated chitosan wafers have indicated that the hydration capacity, mucoadhesion properties and release characteristics are influenced by the annealing process. The annealing step in the freeze-drying cycle helped to obtain wafers with the advantage of enhanced mucoadhesion properties, hydration capacity and consequently drug release property. The final freeze-dried product was an elegant, mechanically strong cake and is expected to exhibit long-term storage stability as the secondary structure of BSA was found to be conformationally stable. The principal implication of these findings is the

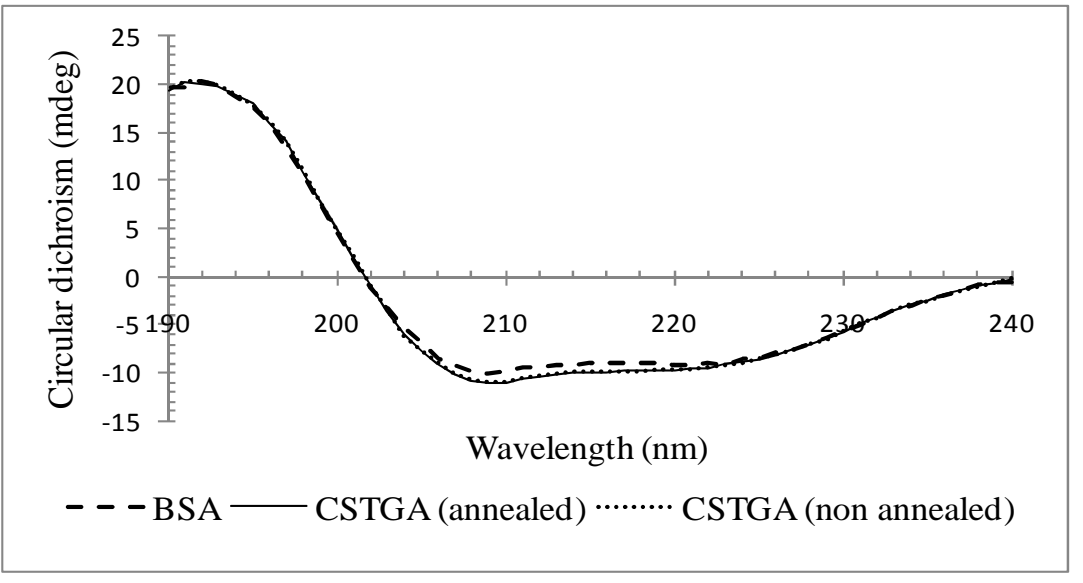

Fig. 5: CD Spectra of native BSA, annealed and non-annealed CSTGA-BSA wafers 
potential application of the novel freeze-dried system for the delivery of proteins via the buccal mucosa.

\section{ACKNOWLEDGEMENT}

This research is being supported by the Commonwealth Scholarship Commission, UK. The authors are grateful to Dr Ian Slipper for the SEM images.

\section{REFERENCES}

Agnihotri, S. A., Mallikarjuna, N. N. and Aminabhavi, T. M. (2004). "Recent advances on chitosan-based micro- and nano-particles in drug delivery." Journal of Controlled Release, 100: 5-28.

Andrew, G. P., Laverty, T. P. and Jones, D. S. (2009). "Mucoadhesive polymeric platforms for controlled drug delivery." European Journal of Pharmaceutics and Biopharmaceutics, 71: 505-518.

Ayensu, I., Mitchell, J. C. and Boateng, J. S. (2011). "Development and physicomechanical characterisation of lyophilised chitosan wafers as potential protein drug delivery systems via the buccal mucosa." Colloids and Surfaces B: Biointerfaces DOI:10.1016/j.colsurfb.2011.11.004

Bernkop-Schnurch, A., Guggi, D. and Pinter Y. (2004). "Thiolated chitosans: development and in vitro evaluation of a mucoadhesive, permeation enhancing oral drug delivery system." Journal of Controlled Release, 94: 177 $-186$.

Bunte, H., Drooge, D. J., Ottjes, G., Roukema, R., Verrijk, R. and Yessine, M. (2010). "Key considerations when developing freeze-dried formulation and current trends." Pharmaceutical Technology Europe Digital, 22: 2-4.

De Campos, A. M., Diebold, Y., Carvalho, E. L., Sanchez, A. and Alonso, M. J. (2004). "Chitosan nanoparticles as new ocular drug delivery systems: in vitro stability, in vivo fate, and cellular toxicity." Pharmaceutical Research, 21: 803-810.

Ernst, O. and Zor, T. (2010). "Linearization of the Bradford Protein Assay." Journal of Visualized Experiments DOI: 10.3791/1918. 1-6.

Giunchedi, P., Juliano, C., Gavini, E., Cossu, M. and Sorrenti, M. (2002). "Formulation and in vivo evaluation of chlorhexidine buccal tablets prepared using drug-loaded chitosan microspheres." European Journal Pharmaceutics and Biopharmaceuticals, 53: 233239.

Hornof, M. D., Kast, C. E. and BernkopSchnurch, A. (2003). "In vitro evaluation of the viscoelastic properties of chitosanthioglycolic acid conjugates." European Journal of Pharmaceutics and Biopharmaceutics, 55: 185-190.

Kelly, S. M., Jess, T. J. and Price, N. C. (2005). "How to study proteins by circular dichroism." Biochimica et Biophysica Acta, 1751: $119-139$.

Liao, X., Krishnamurthy, R. and Suryanarayanan, R. (2007). "Influence of processing conditions on the physical state of mannitol implications in freeze-drying." Pharmaceutical Research, 370-376.

Nochos, A., Douroumis, D. and Bouropoulos, N. (2008). "In vitro release of bovine serum albumin from alginate/HPMC hydrogel beads." Carbohydrate Polymers, 74: 451457.

Peppas, N. A. and Bury, P. A. (1985). "Surface interfacial and molecular aspects of polymer bioadhesion on soft tissues." Journal of Controlled Release, 2: 257-275.

Quiming, N. S., Vergel, R. B., Nicolas, M. G. and Villanueva, J. A. (2005). "Interaction of 
Bovine Serum Albumin and Metallothionein." Journal of Health Science, 51: 8 $-15$

Read, R. C., Naylor, S. C., Potter, C. W., Bond, J., Jabbal-Gill., I., Fisher, A., Illum, L. and Jennings, R. (2005). "Effective nasal influenza vaccine delivery using chitosan." Vaccine, 23: 4367-4374.

Saboktakin, M. R., Tabatabaie, R. M., Maharramov, A. and Ramazanov, M. A. (2011). "Development and in vitro evaluation of thiolated chitosan-Poly (methacrylic-acid) nanoparticles as a local mucoadhesive delivery system." International Journal of Biological Macromolecules, 48: 403-407.

Searles, J. A., Carpenter, J. F. and Randolph, T. W. (2001a). "Annealing to optimize the primary drying rate, reduce freezing-induced drying rate heterogeneity, and determine $\mathrm{T}$ (g)' in pharmaceutical freeze-drying." Journal of Pharmaceutical Sciences, 90: 872887.

Searles, J. A., Carpenter J. F. and Randolph, T. W. (2001b). "The ice nucleation temperature determines the primary drying rate of freezedrying for samples frozen on a temperaturecontrolled shelf'. Journal of Pharmaceutical Sciences, 90: 860-871.
Solomonidou, D., Cremer, K., Krumme, M. and Kreuter, J. (2001). "Effect of carbomer concentration and degree of neutralization on the mucoadhesive properties of polymer films". Journal of Biomaterials and Scientific Polymers, 12: 1191-1205.

Sreenivas, S. A. and Pai, K. V. (2009). "Synthesis of Thiolated Chitosans: promising polymers for prolonged ducoadhesive drug delivery." International Journal of Pharmtech Research, 1(3): 670-678.

Van Der Lubben, I. M., Verhoef, J. C., Borchard, G. and Junginger, H. E., (2001). "Chitosan and its derivatives in mucosal drug and vaccine delivery." European Journal of Pharmaceutical Sciences, 14: 201-207

Vandevord, P. J., Matthew, H. W., Desilva, S. P., Mayton, L., Wu, B. and Wooley, P. H. (2002). "Evaluation of the biocompatibility of a chitosan scaffold in mice". Journal of Biomedical Materials and Research, 59: 585 $-590$

Wu, Z. M., Zhang, X. G., Zheng, C. Li, C. X., Zhang, S. M., Donga, R. N. and Yu, D. M. (2009). "Disulfide-crosslinked chitosan hydrogel for cell viability and controlled protein release." European Journal of Pharmaceutical Sciences, 37: 198-206. 